\title{
Experimental and Theoretical Studies of Transport through Large Scale, Partially Aligned Arrays of Single-Walled Carbon Nanotubes in Thin Film Type Transistors
}

2007

Vol. 7, No. 5

1195-1202

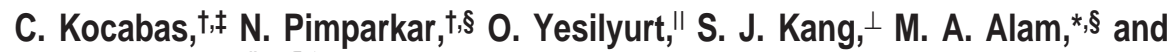 \\ J. A. Rogers ${ }^{\star}, \perp, \#,+, \eta, \diamond$
}

Departments of Physics, Materials and Science Engineering, Chemistry, and Electrical and Computer Engineering, Beckman Institute, and Frederick Seitz Materials Research Laboratory, University of Illinois, Urbana-Champaign 61801, School of Electrical and Computer Engineering, Purdue University, West Lafayette, Indiana 47907-1285, and Department of Materials Science and Nanotechnology, Bilkent University, 06800 Bilkent, Ankara, Turkey

Received December 11, 2006; Revised Manuscript Received March 7, 2007

\begin{abstract}
Gate-modulated transport through partially aligned films of single-walled carbon nanotubes (SWNTs) in thin film type transistor structures are studied experimentally and theoretically. Measurements are reported on SWNTs grown by chemical vapor deposition with systematically varying degrees of alignment and coverage in transistors with a range of channel lengths and orientations perpendicular and parallel to the direction of alignment. A first principles stick-percolation-based transport model provides a simple, yet quantitative framework to interpret the sometimes counterintuitive transport parameters measured in these devices. The results highlight, for example, the dramatic influence of small degrees of SWNT misalignment on transistor performance and imply that coverage and alignment are correlated phenomena and therefore should be simultaneously optimized. The transport characteristics reflect heterogeneity in the underlying anisotropic metal-semiconductor stick-percolating network and cannot be reproduced by classical transport models.
\end{abstract}

Thin films of single-walled carbon nanotubes (SWNTs) represent a class of electronic material that can serve as highperformance semiconducting and/or conducting layers in thin film type field effect transistors (TFT) and other devices. ${ }^{1-8}$ The favorable statistics of such films may provide a route to practical tube-based electronics systems by obviating the need for precise control over the properties or positions of individual SWNTs. The films can consist of any arrangement of tubes, from random networks to perfectly aligned arrays

* Corresponding authors. E-mail: jrogers@uiuc.edu; email alam@ purdue.edu.

$\dagger$ These authors have contributed equally to this work.

Department of Physics, University of Illinois.

\$ School of Electrical and Computer Engineering, Purdue University.

"Department of Materials Science and Nanotechnology, Bilkent University.

${ }^{\perp}$ Department of Materials and Science Engineering, University of Illinois.

\# Department of Chemistry, University of Illinois.

+ Department of Electrical and Computer Engineering, University of Illinois.

II Beckman Institute, University of Illinois.

${ }^{\diamond}$ Frederick Seitz Materials Research Laboratory, University of Illinois. typically in coverages that correspond to somewhat less or somewhat more than a monolayer (appropriate for maximum gate control). In such network TFTs, tube-tube contact resistances have detrimental effects on the overall transistor performance. Reducing or eliminating such tube-tube contacts can improve the performance. This goal can be achieved by designing the devices such that (i) the SWNTs are sufficiently long (length $L_{\mathrm{S}}$ ) to directly bridge the source/ drain electrodes (channel length $L_{\mathrm{C}}$ ) and (ii) the SWNT are configured into dense, aligned arrays that avoid tube-tube overlaps. Generating well-aligned arrays with near-monolayer coverage represents a considerable experimental challenge. Some promising methods rely on chemical vapor deposition growth guided by favorable van der Waals and/or step edge interactions between the tubes and the underlying substrate (quartz or sapphire). ${ }^{9-11}$ When implemented with annealed substrates, moderate catalyst concentrations, and optimized growth conditions, well-aligned arrays at reasonably high coverage are possible. ${ }^{12}$ Even in these cases, however, some 


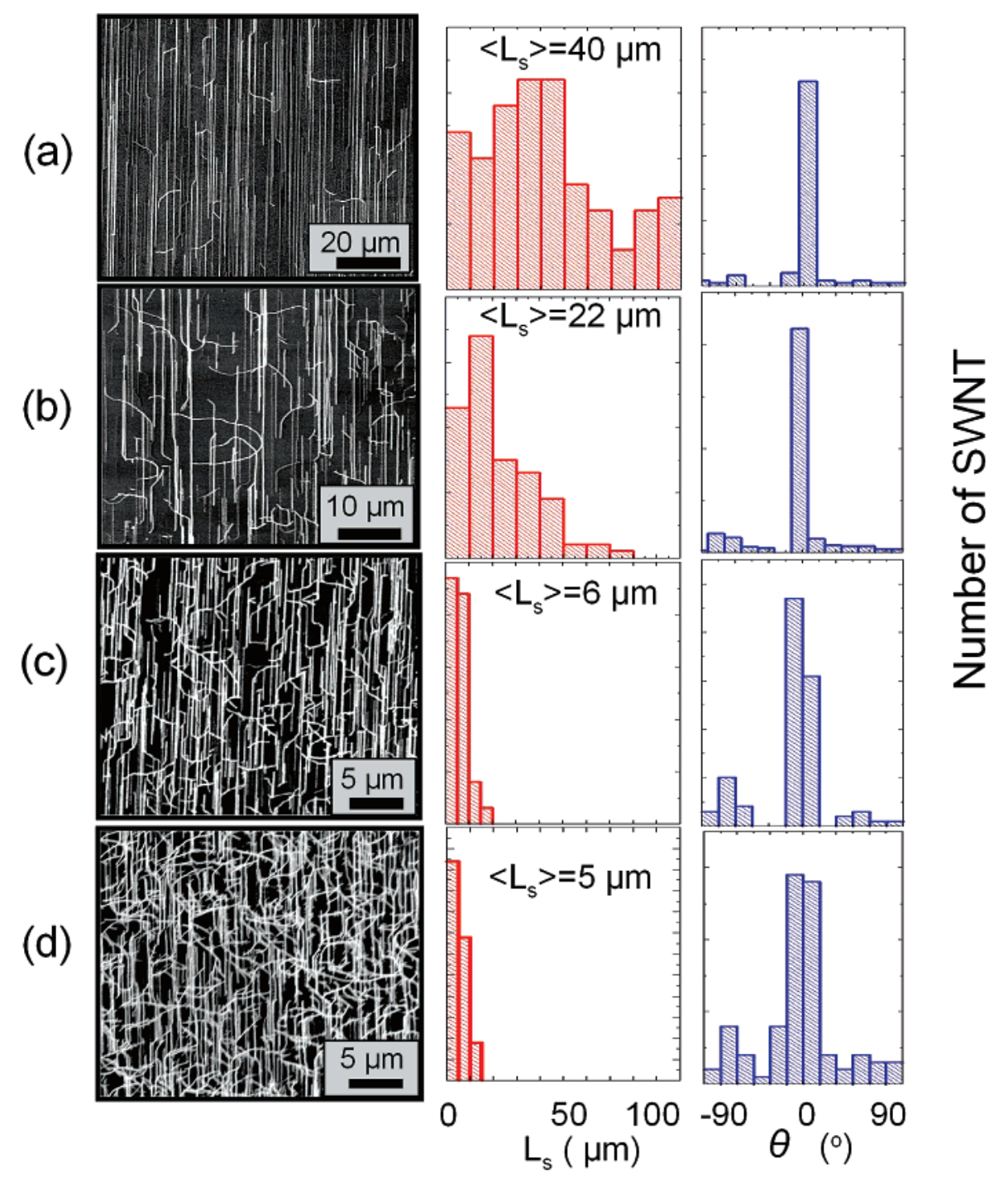

Figure 1. $(\mathrm{a}-\mathrm{d})$ Scanning electron micrographs (SEMs) and statistical information on the four types of films of single-walled carbon nanotubes that were studied. The films range from well aligned, low coverage (a) to partially aligned, high coverage (d) cases. The histogram plots show data for the tube length, $L_{\mathrm{S}}$, and tube orientation, $\theta$, determined from image analysis of the SEMs. The SWNTs in all cases have diameters between 1 and $3 \mathrm{~nm}$.

degree of misalignment exists, which tends to increase with increasing tube coverage. The influence of even a small number of misaligned tubes on transport can be significant, since such tubes can intersect and thereby establish electrical contacts to many other aligned tubes.

Predictive knowledge of the physics of percolative transport through such partially aligned heterogeneous (one-third of the tubes are metallic) arrays, therefore, is a prerequisite to interpret and optimize the electrical performance of these thin films. The system involves, in general, a combination of (i) transport from one electrode to another through individual tubes that bridge the source/drain electrodes and (ii) percolation type transport in an anisotropic tube network. The length and coverage of the tubes and the orientation and length of the transistor channel $\left(L_{\mathrm{C}}\right)$ determine the relative contributions of these two pathways to overall transport in a particular device. Effects of anisotropy in the percolation through purely conductive networks have been studied in structured metal films ${ }^{13}$ and carbon nanotube/ polymer composites. ${ }^{14}$ The present report examines a dif- ferent type of anisotropic network, which involves both gatemodulated percolation and direct transport in transistor structures. This particular problem, which is of paramount importance to the implementation of SWNTs as active layers in thin film electronic systems, as well as the more general case of gate modulated percolation transport in heterogeneous anisotropic networks (i.e., one with both metallic and semiconducting elements), has not been studied before. The results presented here include experimental and theoretical studies of transport and scaling properties of transistors that use submonolayer films of SWNTs with systematically varying degrees of alignment and coverage, from lowcoverage well-aligned films to high-coverage partially aligned ones. Good agreement between measurement and theory validates the key aspects of the model and reveals new routes to improve the transport properties of the films.

Figure 1 shows scanning electron micrographs (SEMs) of SWNT films typical of those studied here. These samples were grown by conventional chemical vapor deposition on ST cut quartz substrates ${ }^{12}$ using spin cast ferritin catalysts. 
Before the growth, the quartz wafers were annealed for $8 \mathrm{~h}$ at $900{ }^{\circ} \mathrm{C}$ in air and cleaned with acetone, isopropyl alcohol, and deionized (DI) water. The annealing conditions, the catalyst concentrations, and the growth conditions determine the coverage and alignment of SWNTs in the resulting films. By varying these parameters, we could achieve films ranging from well to moderately aligned, and from low $(\sim 0.1 \%)$ to high $(\sim 2 \%)$, in terms of the percentage of the surface covered by SWNTs. For the cases studied here, we used four different films (Figure 1) with degrees of alignment defined primarily by the catalyst concentrations. Solutions of the catalyst (ferritin, Aldrich, diluted with DI water) with concentrations of $0.1,0.2,0.76$, or $3.8 \mathrm{mg} / \mathrm{mL}$ were placed on the quartz substrate, and after $1 \mathrm{~min}$, a few methanol droplets were added. The substrate was then rotated at $3000 \mathrm{rpm}$ for $30 \mathrm{~s}$ using a spin coater, followed by thorough rinsing with methanol. Heating at $900{ }^{\circ} \mathrm{C}$ for 10 min oxidized the catalyst. Cooling to room temperature and then heating to $900{ }^{\circ} \mathrm{C}$ in a hydrogen environment reduced the catalyst. Purging with hydrogen at $900{ }^{\circ} \mathrm{C}$ for $1 \mathrm{~min}$ and then introducing a flow of methane $(2500 \mathrm{sccm})$ and hydrogen $(75 \mathrm{sccm})$ at $900{ }^{\circ} \mathrm{C}$ for $10 \mathrm{~min}$ led to the growth of SWNTs. The diameters of the tubes in all cases are between 1 and $3 \mathrm{~nm}$, as determined by atomic force microscopy, ${ }^{9}$ with some trend toward larger diameter tubes (or small bundles) with increasing coverage. Past studies of SWNTs grown by chemical vapor deposition with ferritin catalysts indicate that roughly one-third of the tubes are metallic and two-thirds are semiconducting..$^{15}$ The ratio of numbers of semiconductor to metallic tubes was evaluated directly by electrical burning of a single or a few tubes and counting the metallic and semiconducting tubes individually. Details of the experiments are given in Supporting Information. The histogram plots summarize the distributions of tube lengths $\left(L_{S}\right)$ and orientations $(\theta)$, as determined from image analysis of the SEMs. The orientations of the tubes often change at one or both of their ends. The reported values of $\theta$ correspond to segments of tubes that have a single orientation, as observed in the field of view. Similarly, the values of $L_{\mathrm{S}}$ correspond to the lengths of these tube segments. The average tube lengths, $\left\langle L_{S}\right\rangle$, were determined by the arithmetic mean of the measured $L_{\mathrm{S}}$ values; the lengths for films $1-4$ were $40,22,6$, and $5 \mu \mathrm{m}$, respectively. The degree of alignment can be defined in terms of an anisotropy parameter, $R$, where $R=L_{\mid} / L_{\perp}=\sum_{i=1}{ }^{N} \mid L_{\mathrm{S}, i}$ $\cos \theta_{i}\left|/ \sum_{i=1}^{N}\right| L_{\mathrm{S}, i} \sin \theta_{i} \mid$. Films $1-4$ have $R=21.4,6.5,6.0$, and 2.9, respectively. These growth procedures yield strong correlations between the coverage, the tube length, and the degree of anisotropy. We also note that while the approaches presented here require quartz substrates, the anisotropic networks can be transferred to other substrates, including thin, flexible plastic sheets. ${ }^{16}$

We constructed top gate transistors in which the films (films 1-4, as illustrated in Figure 1) serve as the semiconductor layer. The fabrication began with growth of the films on quartz, followed by definition of source and drain electrodes of Ti/Pd $(1 \mathrm{~nm} / 25 \mathrm{~nm}$; deposited by electron beam evaporation) by photolithography and liftoff. A spin cast layer of a photocurable epoxy (MicroChem, SU8-2) with thickness $\sim 1.3 \mu \mathrm{m}$ provided a gate dielectric with dielectric constant of $\epsilon_{\mathrm{r}}=4.0$ and per unit area capacitance of 2.8 $\mathrm{nF} / \mathrm{cm}^{2}$. We chose a thick polymer for the present studies for three reasons: (1) these materials enable simple, highyield fabrication of devices with very low gate leakage, (2) the polymers, unlike many of the thin high-capacitance inorganic materials that have been explored in single tube devices, do not alter the electronic properties of the tubes, and (3) the gate coupling to the tubes can be approximated by a parallel plate model for the capacitance (i.e., the tubetube separations are, in most cases, small compared to the thickness of the gate dielectric). Thinner dielectrics are desirable for device performance but are not important for the present studies. Photolithography and liftoff defined a gate electrode on top of this dielectric, aligned to the channel, and also overlapping parts of the source/drain electrodes. Openings in the dielectric formed by photolithographic patterning provided electrical probing access to the source and drain. We used these procedures to fabricate the transistors with channel lengths of $L_{\mathrm{C}}=5,10,25,50$ and $100 \mu \mathrm{m}$ all with widths of $W=250 \mu \mathrm{m}$. Figure 2 shows the device geometry. The channels were oriented either parallel or perpendicular to the preferred direction for tube alignment. For each device, currents between source and drain were measured at a bias $\left(V_{\mathrm{sd}}\right)$ of $0.5 \mathrm{~V}$ while sweeping the gate $\left(V_{\mathrm{g}}\right)$ between $-40 \mathrm{~V}$ and $40 \mathrm{~V}$. In all cases the current to the gate was less than $100 \mathrm{nA}$.

To analyze the transport characteristics of these films, we consider the scaling behavior of "ON" and "OFF" currents, $I_{\mathrm{on}}$ and $I_{\mathrm{off}}$, as a function of channel length $L_{\mathrm{C}}$. The ON current is measured at $V_{\mathrm{sd}}=0.5 \mathrm{~V}$ and $V_{\mathrm{g}}=-40 \mathrm{~V}$; the OFF current corresponds to the minimum current observed for the range of gate voltages between $-40 \mathrm{~V}$ and $40 \mathrm{~V}$, with $V_{\text {sd }}=0.5 \mathrm{~V}$. (The slight ambipolar response of the transistors motivates the use of such a definition for $I_{\text {off. }}$ ) Although the transistors exhibited some hysteresis, as is typical for devices of this type, the current values, $I_{\mathrm{on}}$ and $I_{\text {off, }}$ which form the focus of this paper, remain robust, independent of direction or history of applied voltages to the gate. Figure 2e shows a typical device response and channel length dependence. A total of 80 devices were measured for each of the films 1-4: 40 with the channel along the preferred alignment direction and 40 with orientations perpendicular to it for five different channel lengths, $L_{\mathrm{C}}=5,10,25,50$ and $100 \mu \mathrm{m}$.

Figure 3 illustrates a representative set of different devices that were studied. The layouts of the tubes have correlated levels of coverage, alignment, and tube length, for the growth procedures used here. (Recent reports describe growth strategies that eliminate some of these correlations. ${ }^{17}$ ) At low coverage, extremely high degrees of alignments and long tube lengths result, leading to many tubes that bridge the source and drain electrodes, with almost no tube-tube overlaps. This configuration leads to large ON currents, but also large OFF currents due to the presence of the metallic tubes. At high coverage, the alignment can be poor and the tubes are short; this configuration leads to percolation 
(a)

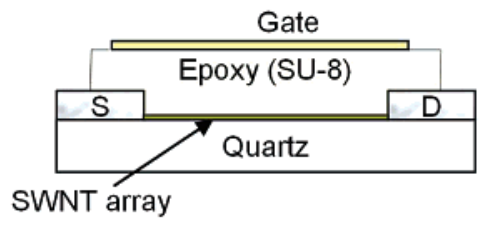

(d)

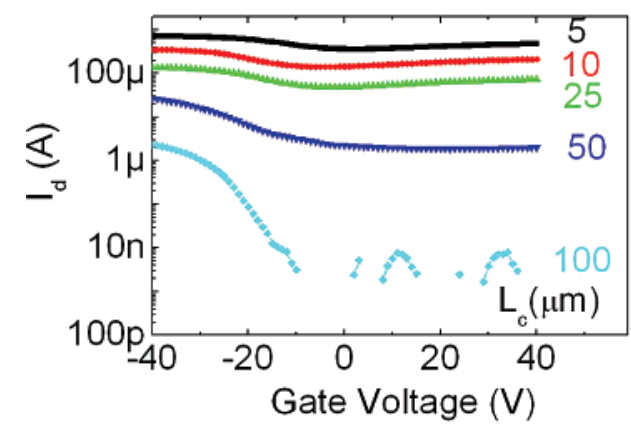

(b)

(e)
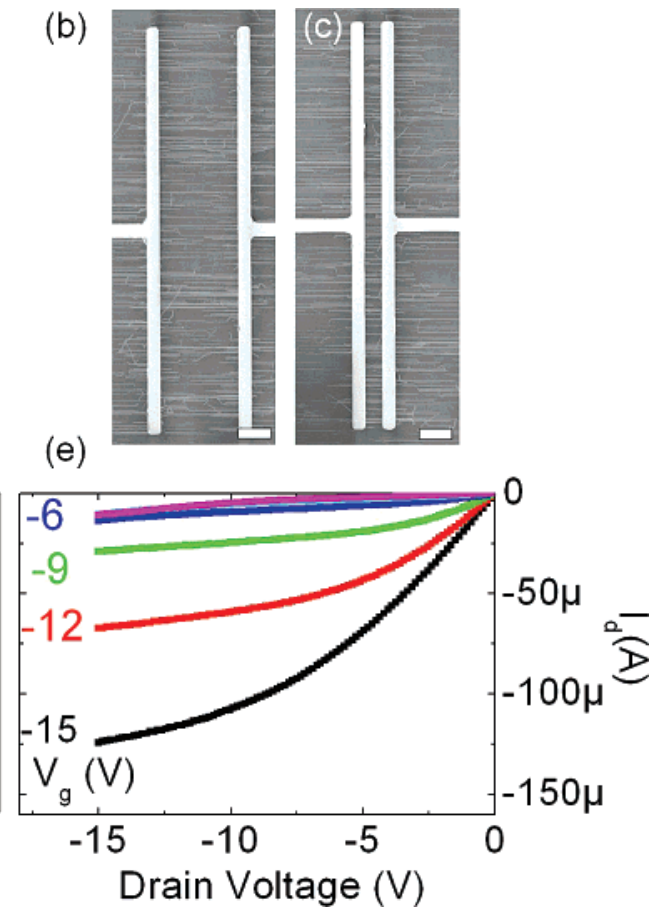

Figure 2. (a) Device layout of thin film transistors fabricated on SWNT. (b, c) SEM images of S/D electrodes with SWNTs. Scale bar is $10 \mu \mathrm{m}$. Typical data collected from representative devices that reveal scaling properties of transport as a function of transistor channel length, $L_{\mathrm{C}}$ of $5,10,25,50$, and $100 \mu \mathrm{m}$ (d) and output characteristics for $100 \mu \mathrm{m}$ channel length (e).

transport pathways and high on/off ratios due to the small chance of bridging tubes or purely metallic current pathways. Similar but different types of considerations lead to different behaviors with different channel orientations. Parts $\mathrm{c}$ and $\mathrm{d}$ of Figure 3 show devices with same tube coverage but different channel orientations. Lower ON currents and higher on/off ratios are observed for perpendicular orientation (Figure 3f) compared to the parallel orientation. Measurements of this type provide a means to quantify the level of electrical anisotropy in the network, in terms of the ratio of ON currents measured in the parallel and perpendicular directions. Figure $3 g$ shows the calculated current anisotropy, $A=I_{||} / I_{\perp}$, evaluated in the on state, of aligned, dense partially aligned, and random network cases. The highest anisotropy occurs, as expected, in the aligned devices. Increasing tube coverage tends to lower the anisotropy. In cases that involve some level of percolation transport pathways, the ON current anisotropy decreases with increasing channel length. Completely random network grown, for example on amorphous $\mathrm{SiO}_{2}$, does not provide any anisotropic conduction.

A review of the measured electrical characteristics for aligned (Figures 1a and 4a), partially aligned (Figures 1c and $4 \mathrm{~b}$ ), and dense partially aligned (Figures $1 \mathrm{~d}$ and $4 \mathrm{c}$ ) networks shows that, analogous to classical transistors, $I_{\text {on }}$ and $I_{\text {off }}$ decrease with increasing $L_{\mathrm{C}}$. The corresponding insets show samples of randomly generated networks. Unlike classical transistors, however, the (channel-length) scaling exponents of $I_{\text {on }}$ and $I_{\text {off }}$, are significantly different., ${ }^{7,18}$ This anomaly is highlighted in the ratio of $I_{\mathrm{on}} / I_{\text {off }}\left(=R_{\mathrm{I}}\right)$ which, instead of remaining constant (as would be expected for classical transistors), increases with $L_{C}$. Moreover, the rate at which $R_{\mathrm{I}}$ increases with $L_{\mathrm{C}}$ increases with decreasing coverage. Finally, one anticipates the $I_{\mathrm{on}}$ and $I_{\text {off }}$ to individually approach zero for perfectly aligned network as $L_{\mathrm{C}} \rightarrow$ $L_{S}$, yet remarkably both currents remain finite even with $L_{\mathrm{C}}$ $\gg L_{\mathrm{S}}$. These three counterintuitive transport characteristics of aligned tubes indicate that classical "top-down" mobilitybased models are no longer adequate and that a "bottomup" 18 treatment of transport based on stick-percolation models is required.

We construct a numerical stick-percolation model for this system by randomly populating a two-dimensional (2D) grid by sticks of length $\left(L_{S}\right)$ and orientation $(\theta)$ with probability density function (PDF) consistent with experimental conditions (Figure 1). One-third of the generated tubes are metallic and the remaining two-third are semiconducting (Supporting information) (the results are not too sensitive to the precise fraction of metallic tubes between $1 / 3$ and $1 / 4$, see Supporting Information). Since, $L_{\mathrm{C}}$ and $L_{\mathrm{S}}$ are much larger than the phonon mean free path, contact resistances are not important and linear-response transport (small $V_{\text {sd }}$ and constant $V_{\mathrm{g}}$ obviates the need to solve the Poisson equation) within individual stick segments of this anisotropic stick-network system is well described by drift-diffusion theory. ${ }^{7,19-21}$ The low bias drift-diffusion equation, $J=q \mu n \mathrm{~d} \varphi / \mathrm{d} s$, when combined with current continuity equation, $\mathrm{d} J / \mathrm{d} s=0$, gives the nondimensional potential $\varphi_{i}$ along tube $i$ as (see Supporting Information)

$$
\mathrm{d}^{2} \varphi_{i} / \mathrm{d} s^{2}-c_{i j}\left(\varphi_{i}-\varphi_{j}\right)=0
$$

Here, $s$ is the length along the tube and $c_{i j}=G_{0} / G_{1}$ is the dimensionless charge-transfer coefficient between tubes $i$ and 

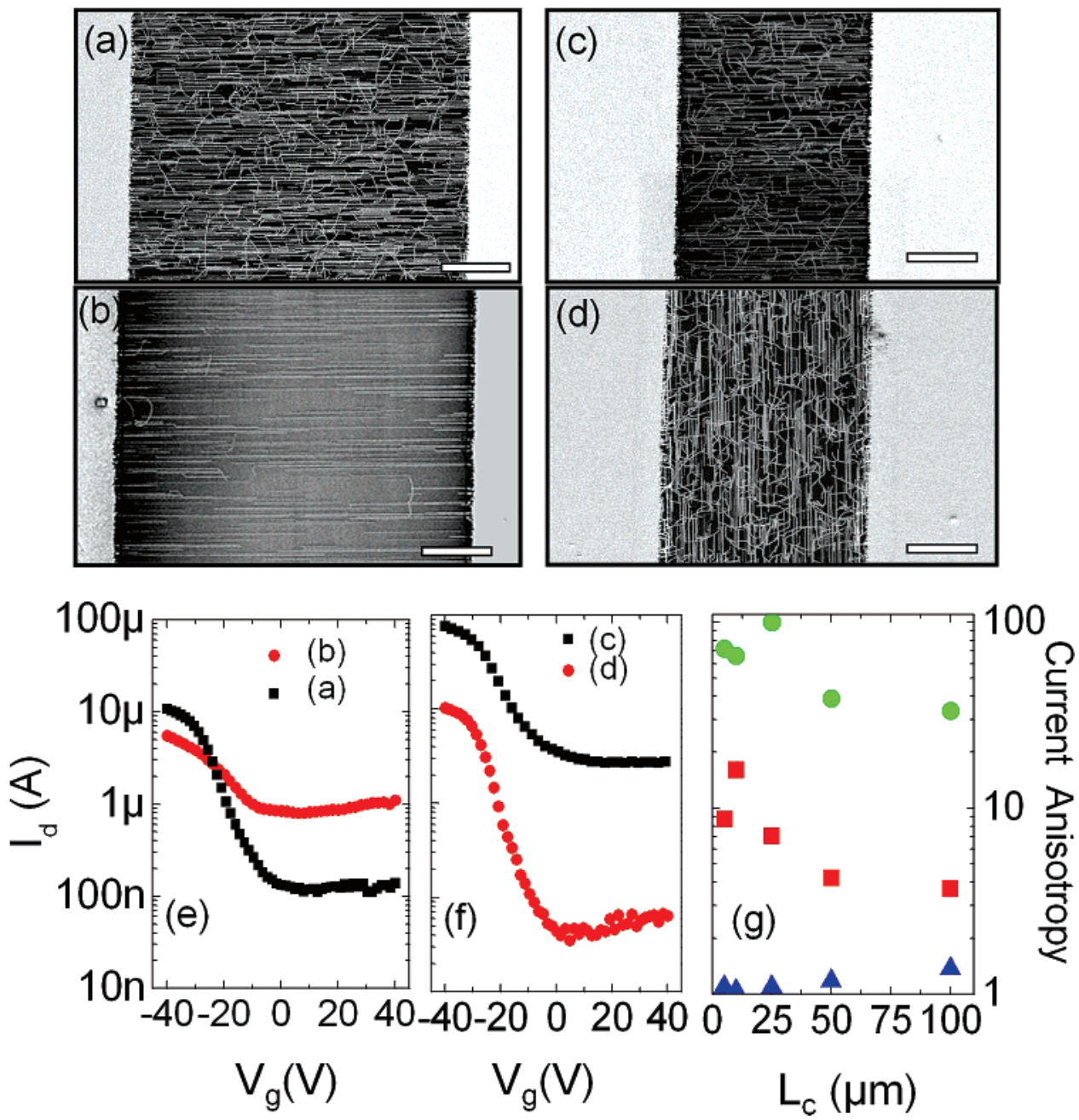

Figure 3. Scanning electron micrographs (SEMs) of transistors with channel lengths of $50 \mu \mathrm{m}(\mathrm{a}, \mathrm{b})$ and $25 \mu \mathrm{m}$ (c, d) with different channel orientations and degrees of alignment and coverage. The scale bars are $10 \mu \mathrm{m}$. (e) Transfer curves of two devices with different tube coverage and the same channel length $\left(L_{\mathrm{C}}=50 \mu \mathrm{m}\right)$. (f) Transfer curves of devices with channels oriented parallel (II, black) and perpendicular $\left(\perp\right.$, red) to the alignment direction. (g) Channel length dependence of ON current anisotropy $\left(I_{\mid} / I_{\perp}\right)$ for aligned (green), dense partially aligned (red), and random network (blue).

$j$ at their intersection point. $G_{0}\left(\sim 0.1 \mathrm{e}^{2} / \mathrm{h}\right)^{22}$ and $G_{1}(=q n \mu /$ $\Delta x)^{7}$ are mutual and self-conductance of the tubes. Here, $n$ is carrier density, $\mu$ is mobility, and $\Delta x$ is grid spacing. The network contains both metallic and semiconducting tubes. $I_{\text {on }}$ is computed by assigning $G_{1}$ (metal) $\sim G_{1}($ semi), while $I_{\text {off }}$ is compute by setting $G_{1}(\mathrm{semi})=10^{-4} \times G_{1}$ (metal) ${ }^{23}$ $G_{1}$ (metal) is assumed to be relatively insensitive to $V_{\mathrm{g}}$. Hundreds of such samples are constructed to accurately reflect the PDF of length and anisotropy distribution in Figure 1 , and the average of these currents is compared to the measured data.

Before we use our stick-percolation model to interpret the three counterintuitive features of anisotropic network discussed above and shown in Figure 4, let us first analyze the simpler case of ON current dependence on $L_{C}$ for the completely random stick network. ${ }^{7}$ As is well-known, in a classical 2D film conductor the current will simply be inversely proportional to $L_{\mathrm{C}}$, i.e., $I_{\mathrm{on}} \sim k / L_{\mathrm{C}}$, which is Ohm's law. Here, $k$ is a material-specific constant. However, stick networks are nonclassical 2D conductor and satisfy the finite size scaling relationship ${ }^{24,25}$

$$
I_{\text {on }} \sim \frac{k}{L_{\mathrm{S}}}\left(\frac{L_{\mathrm{S}}}{L_{\mathrm{C}}}\right)^{m}
$$

where, the current exponent $m$ is universal constant that depends only on the normalized coverage $\left(\rho_{\mathrm{S}} L_{\mathrm{S}}{ }^{2}, \rho_{\mathrm{S}}\right.$ is the number of SWNTs in unit area and $L_{\mathrm{s}}$ is the length of the $S W N T$ ) and the anisotropy of the tubes. For example, for completely random network ${ }^{24}$ and at coverage much higher than the percolation threshold $\left(\rho_{\mathrm{S}} L_{\mathrm{S}}{ }^{2} \gg 4.236^{2} / \pi\right),{ }^{26}$ most of the sticks in the network are connected and take part in conduction as shown in Figure 5a. Therefore, the high coverage network behaves as a $2 \mathrm{D}$ conductor and $m \sim 1$, i.e., $I_{\mathrm{on}} \sim k / L_{\mathrm{C}}$ (Figure $5 \mathrm{c}$, red circles are experimental values and the red line is the simulation), which is again Ohm's law. But for a random network with coverage at/near the percolation threshold $\left(\rho_{\mathrm{S}} L_{\mathrm{S}}{ }^{2} \sim 4.236^{2} / \pi\right)$ all the sticks are not connected to source and drain and there are many islands or pools of unconnected sticks. These pools of sticks form new percolating paths as $L_{\mathrm{C}}$ is reduced-effectively increasing the width of the channel and increasing the current exponent to $m=1.9$, $^{7,24}$ i.e., $I_{\text {on }} \sim\left(k / L_{\mathrm{S}}\right)\left(L_{\mathrm{S}} / L_{\mathrm{C}}\right)^{1.9}$. For example, the 


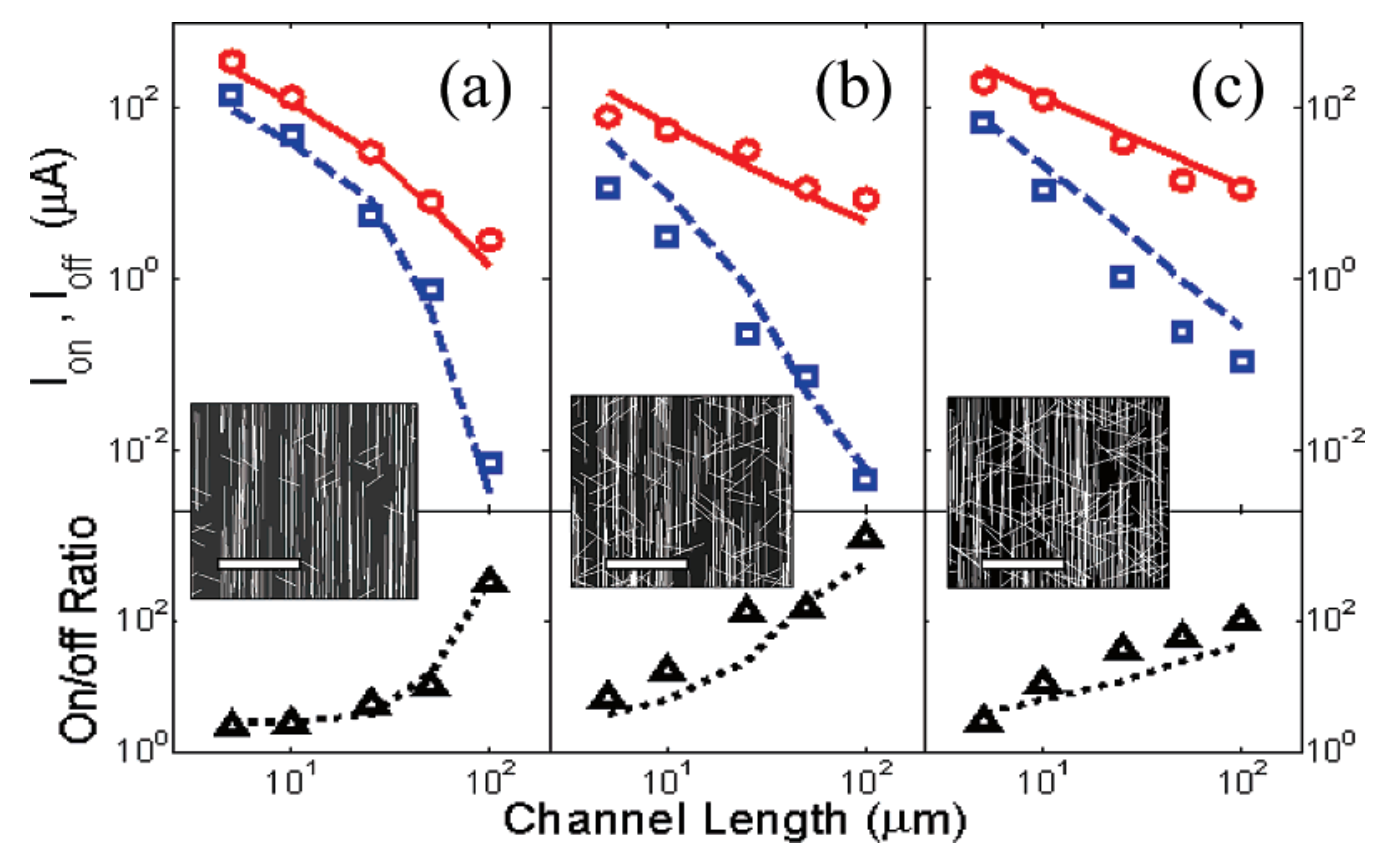

Figure 4. ON current $I_{\mathrm{on}}$, OFF current $I_{\text {off }}$, and on/off ratio for (a) aligned, (b) partially aligned, and (c) dense partially aligned networks corresponding to panels a, c, and d of Figure 1, respectively, where the symbols show experimental results and lines show simulation results. The current exponents, $m_{\mathrm{R}}$ (eq 1) for (a), (b), and (c) are given by, $m_{\mathrm{on}}=1.7, \sim 1$, and $\sim 1, m_{\text {off }}=4.8,2.78$, and 1.53, and the exponents for the on/off ratio $=-3.1,-1.78,-0.53$, respectively. The insets show the simulated networks with scale bar $=10 \mu \mathrm{m}$.

intermediate coverage network (density above the percolation threshold, but still low compared to the dense network) shown in Figure 5b, has a current exponent of $m=1.4$. The dotted lines show the current paths in each device. The ON current scaling appears in Figure 5c (blue squares). Figure $5 \mathrm{~d}$ shows the current exponent $(m)$ vs coverage, where the symbols represent the two curves in Figure 5c. Devices with high coverage and long channel length $\left(L_{\mathrm{C}} \gg L_{\mathrm{S}}\right)$ approach the macroscopic device limit (classical ohmic behavior). ${ }^{5,27}$ Here, the random networks with high coverage (Figure 5a, $\rho_{\mathrm{S}} L_{\mathrm{S}}{ }^{2} \sim 20-40$ ) and low coverage (Figure $5 \mathrm{~b}, \rho_{\mathrm{S}} L_{\mathrm{S}}{ }^{2} \sim 5-10$ ) were grown on $\mathrm{SiO}_{2}(100 \mathrm{~nm}) / \mathrm{Si}$ where $\mathrm{Si}$ forms a back gate.

Next, let us look at the second puzzle of the on/off ratio: The scaling of the on/off ratio, $R_{\mathrm{I}}$, for random networks, shown in Figure 5e, in the high (red circles) and low (blue squares) coverage cases provides additional insights. Note that approximately one-third of the tubes are metallic and two-third are semiconducting (Supporting information). In the ON state, the total (metallic plus semiconducting) tube coverage $\rho_{\mathrm{S}} L_{\mathrm{S}}{ }^{2}$ determines the experimental current exponent $m_{\mathrm{on}}$ as discussed earlier. On the other hand, the effective coverage of the network at OFF state is $\sim \rho_{\mathrm{S}} L_{\mathrm{S}}{ }^{2} / 3$ (only metallic tubes conduct) with the current exponent being $m_{\text {off- }}$ Since $m$ scales inversely as $\rho_{\mathrm{S}} L_{\mathrm{S}}{ }^{2}$ (Figure $5 \mathrm{~d}$ ), $m_{\mathrm{on}}<m_{\text {off }}$. Since $R_{\mathrm{I}} \equiv I_{\mathrm{on}} / I_{\mathrm{off}} \sim\left(1 / L_{\mathrm{C}}\right)^{m_{\mathrm{on}}} /\left(1 / L_{\mathrm{C}}\right)^{m_{\mathrm{off}}}=\left(1 / L_{\mathrm{C}}\right)^{m_{\mathrm{on}}-m_{\mathrm{off}}}$, therefore $R_{\mathrm{I}}$ increases with the exponent $m_{\mathrm{on}}-m_{\mathrm{off}}$ as a function of $L_{\mathrm{C}}$. Moreover, the model predicts that the $R_{\mathrm{I}}$ vs $L_{\mathrm{C}}$ increases more rapidly for lower coverage network. This is a consequence of rapidly changing exponent, $m$, at lower coverage vs relatively constant $m$ at higher coverage (compare red circles to blue squares in Figure 5e).

The analysis of ON current $\left(I_{\text {on }}\right)$, OFF current $\left(I_{\text {off }}\right)$, and on/off ratio $\left(R_{\mathrm{I}}\right)$ for anisotropic networks (Figure 4) is qualitatively similar to that of the random networks (Figure 5). In fact, the basic explanations of the first two nonintuitive features of an anisotropic network, i.e., (a) nonclassical scaling of ON current and OFF current scaling and (b) increase in $R_{\mathrm{I}}$ with $L_{\mathrm{C}}$ in Figure 4 , are exactly the same as those of the features shown in Figure 5 (just discussed above). However, quantitatively there are significant differences: First note that the high coverage anisotropic networks show exponents $(\sim 1)$ that are similar to the random network (red circles in Figure 4b,c and in Figure 5c). The results are completely different for the lower coverage cases. Because of the tradeoff between tube coverage, tube length, and orientation associated with the growth process, the current exponents can change drastically. The stick percolation model described above can be employed to explain the behavior of these anisotropic networks and the simulations (lines) agree with the experiments (symbols) well as shown in Figure 4. Each simulation point of Figure 4 and 5 reflects the average solution of $\sim 200$ statistical samples. The current exponents for aligned (Figures 1a and 4a), partially aligned (Figures 1c and 4b), and dense partially aligned (Figures 1d and $4 \mathrm{c}$ ) networks for $I_{\text {on }}, I_{\text {off, }}$, and on/off ratio are given by $m_{\mathrm{on}}=1.7, \sim 1, \sim 1, m_{\mathrm{off}}=4.8,2.78,1.53$, and $m_{\mathrm{on}}-m_{\mathrm{off}}=$ $-3.1,-1.78,-0.53$, respectively. Although some expected scaling of current exponents and current outputs can be observed through comparisons between device types, each network case must be analyzed separately due to correlations between tube length, tube alignment, and coverage. For the anisotropic networks, low coverage provides low on/off ratios and high coverage provides on/off ratios similar to random networks. In the former cases, spanning tubes dominate transport, such that the on/off ratio is nearly constant $(<10)$ up to channel length of $50 \mu \mathrm{m}$. Intermediate tube coverage provides the highest on/off ratio. All types of networks yield 
(a)
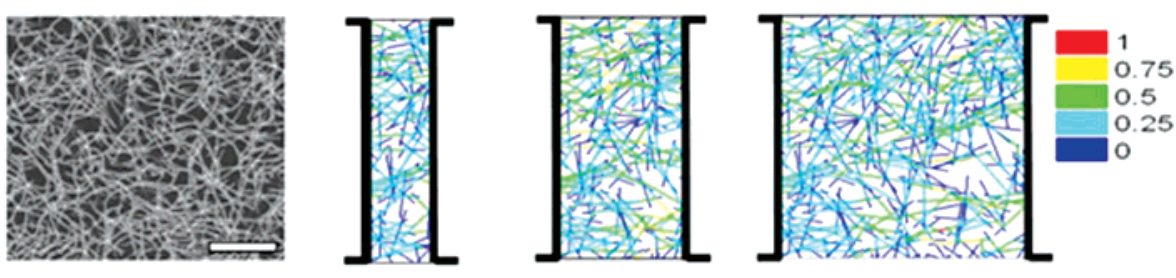

(b)

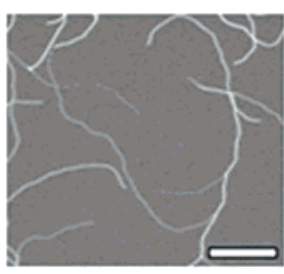

(c)

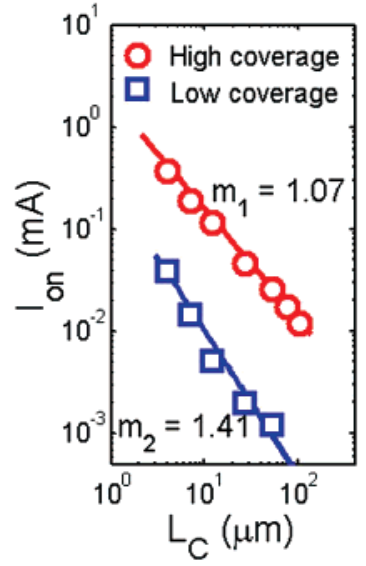

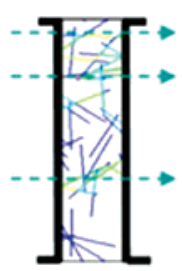

(d)

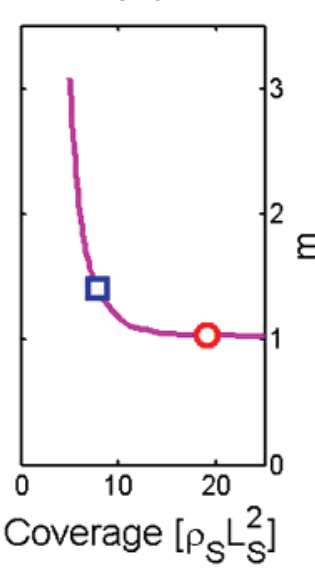

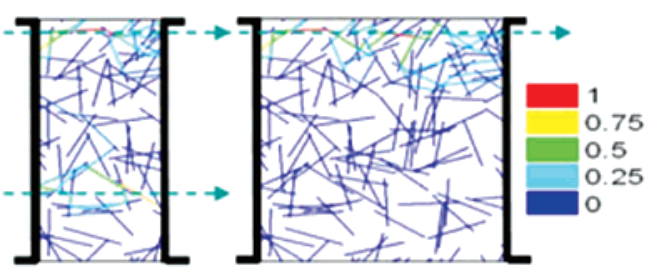

(e)

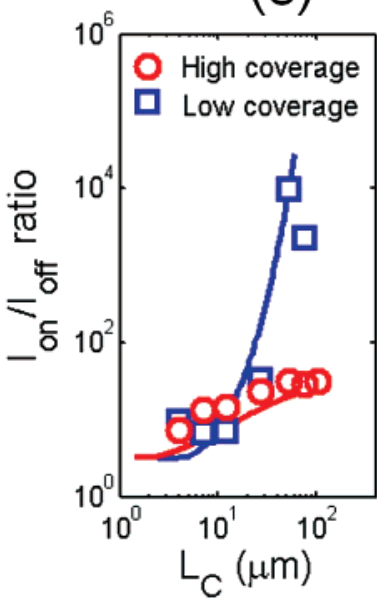

Figure 5. SEM images (scale bar is $5 \mu \mathrm{m}$ ) and simulated normalized current distribution for network with high (a) and low (b) coverage for $L_{\mathrm{C}}=4,8,16 \mu \mathrm{m}$, respectively. The dotted arrows show the current paths. (c) Experimental (symbols) and simulated (lines) ON current $\left(I_{\mathrm{on}}\right)$ vs channel length $\left(L_{\mathrm{C}}\right)$ for high (red circles, $\left.\rho_{\mathrm{S}} L_{\mathrm{S}}{ }^{2} \sim 20\right)$ and low (blue squares $\rho_{\mathrm{S}} L_{\mathrm{S}}{ }^{2} \sim 8$ ) coverage completely random network with current exponent $m_{1}=1.07$, and $m_{2}=1.41$, respectively. (d) Simulated current exponent $(m)$ for random networks with coverage $\left(\rho_{\mathrm{S}} L_{\mathrm{S}}{ }^{2}\right)$ decreases monotonically with increasing $\rho_{S} L_{S}{ }^{2}$ approaching the classical limit of $m=1$ for higher coverage. The points indicated correspond to high (red circle) and low (blue square) coverage curves in c. (e) $I_{\mathrm{on}} / I_{\mathrm{off}}$ ratio vs channel length $\left(L_{\mathrm{C}}\right)$ for same coverage increases with $L_{\mathrm{C}}$ and the rate of increase is more for lower coverage.

lower limits on the on/off ratio given by $1+2 G_{1}(\mathrm{semi}) /$ $G_{1}$ (metal) $\sim 3$ (assuming equal conductance in the ON state) as $L_{C} \rightarrow 0$, where all the tubes bridge S/D directly irrespective of alignment (Figures 4 and 5e).

The stick percolation approach also highlights the importance of misalignment in dictating long-channel transistor performance and resolves the third dilemma so as to why the currents do not drop to zero as $L_{\mathrm{C}}$ approaches $L_{\mathrm{S}}$. In Figure 1a, the degree of misalignment appears negligible. Had this been the case, $I_{\text {on }}$ and $I_{\text {off }}$ would have dropped dramatically as $L_{\mathrm{C}} \rightarrow L_{\mathrm{S}}$, because the percolation threshold for perfectly aligned system approaches infinity $\left(\rho_{\text {perc }} \rightarrow \infty\right)$. Yet, the $I_{\text {on }}$ and $I_{\text {off }}$ currents show no dramatic change as $L_{\mathrm{C}}$ approaches $L_{\mathrm{S}}$. This is because a very small number of short misaligned tubes can bridge the (otherwise unconnected) longer tubes and dramatically reduce $\rho_{\text {perc }}$ to finite values. Misalignment also reduces the variations in current output with orientation of the channel relative to the preferred direction of tube alignment. In the long channel limit $\left(L_{\mathrm{C}}>\right.$ $L_{S}$ ), this anisotropy can be extremely high, since the absence of spanning tubes in the perfectly aligned case yields zero current output (in both the ON and OFF states). For short channels $\left(L_{\mathrm{C}}<L_{S}\right)$, the degree of anisotropy is moderate, with current ratios in the range of $\pi / 2$ for the perfectly aligned and isotropic cases.

A related finding is that our results imply that for transistors with $L_{\mathrm{C}}>L_{S}$, perfect alignment is not optimal and would actually carry less current than slightly nonaligned systems. In this case, percolation dominates and high on/off ratios are possible even with a significant population of metallic tubes. On the other hand, short channel transistors $\left(L_{\mathrm{C}}<L_{\mathrm{S}}\right)$, which are necessary for high-performance technologies, have low on/off ratios, which reflect roughly the relative numbers of metallic and semiconducting tubes. In this regime, an approach for removing the effects of the metallic tubes might be necessary to produce acceptable devices. ${ }^{2,28,29}$ These and other predictions based on these models provide insights into methods to optimize SWNT films for various applications in electronics.

Acknowledgment. We thank S. Kumar and Professor J. Y. Murthy for help with generating random networks and Professor J. L. Gray for the 1D device simulator "ADEPT". We thank Professor Moonsub Shim and Taner Ozel for 
micro-Raman experiments. O.Y. acknowledges financial support from NanoTam (Bilkent University). We also thank T. Banks and K. Colravy for help with processing using facilities at the Frederick Seitz Materials Research Laboratory. This material is based upon work supported by the National Science Foundation under Grant DMI-0328162, the U.S. Department of Energy, Division of Materials Sciences under Award No. DEFG02-91ER45439, through the Frederick Seitz MRL and Center for Microanalysis of Materials at the University of Illinois at Urbana-Champaign, the Network for Computational Nanotechnology, and the Lilly Foundation.

Supporting Information Available: Discussions of determination of percentage of metallic and semiconducting SWNT, derivation of nondimensional potential along the tube, and effect of variation of metallic tube ratio. This material is available free of charge via the Internet at http:// pubs.acs.org.

\section{References}

(1) Snow, E. S.; Novak, J. P.; Campbell, P. M.; Park, D. Appl. Phys. Lett. 2003, 82, 2145-2147.

(2) Zhou, Y. X.; Gaur, A.; Hur, S. H.; Kocabas, C.; Meitl, M. A.; Shim, M.; Rogers, J. A. Nano Lett. 2004, 4, 2031-2035.

(3) Kocabas, C.; Meitl, M. A.; Gaur, A.; Shim, M.; Rogers, J. A. Nano Lett. 2004, 4, 2421-2426.

(4) Meitl, M. A.; Zhou, Y. X.; Gaur, A.; Jeon, S.; Usrey, M. L.; Strano, M. S.; Rogers, J. A. Nano Lett. 2004, 4, 1643-1647.

(5) Hu, L.; Hecht, D. S.; Gruner, G. Nano Lett. 2004, 4, 2513-2517.

(6) Hur, S. H.; Kocabas, C.; Gaur, A.; Park, O. O.; Shim, M.; Rogers, J. A. J. Appl. Phys. 2005, 98, 1143021-1143026.

(7) Kumar, S.; Murthy, J. Y.; Alam, M. A. Phys. Rev. Lett. 2005, 95, $0668021-0668024$.

(8) Hur, S. H.; Khang, D. Y.; Kocabas, C.; Rogers, J. A. Appl. Phys. Lett. 2004, 85, 5730-5732.

(9) Kocabas, C.; Hur, S. H.; Gaur, A.; Meitl, M. A.; Shim, M.; Rogers, J. A. Small 2005, 1, 1110-1116.
(10) Ismach, A.; Segev, L.; Wachtel, E.; Joselevich, E. Angew. Chem., Int. Ed. 2004, 43, 6140-6143.

(11) Han, S.; Liu, X. L.; Zhou, C. W. J. Am. Chem. Soc. 2005, 127, 52945295.

(12) Kocabas, C.; Shim, M.; Rogers, J. A. J. Am. Chem. Soc. 2006, 128, $4540-4541$.

(13) Smith, L. N.; Lobb, C. J. Phys. Rev. B 1979, 20, 3653-3658.

(14) Du, F. M.; Fischer, J. E.; Winey, K. I. Phys. Rev. B 2005, 72, 1214041-1214044.

(15) Li, Y. M.; Peng, S.; Mann, D.; Cao, J.; Tu, R.; Cho, K. J.; Dai, H. J. J. Phys. Chem. B 2005, 109, 6968-6971.

(16) Hur, S. H.; Park, O. O.; Rogers, J. A. Appl. Phys. Lett. 2005, 86, 2435021-2435023.

(17) Kang, S. J.; Kocabas, C.; Ozel, T.; Shim, M.; Pimparkar, N.; Alam, M. A.; Rogers, J. A. Nat. Nanotechnol., in press.

(18) Pimparkar, N.; Guo, J.; Alam, M. A. IEEE Trans. Electron Devices, in press.

(19) Li, S. D.; Yu, Z.; Rutherglen, C.; Burke, P. J. Nano Lett. 2004, 4, 2003-2007.

(20) Perebeinos, V.; Tersoff, J.; Avouris, P. Nano Lett. 2006, 6, 205208.

(21) Zhou, X. J.; Park, J. Y.; Huang, S. M.; Liu, J.; McEuen, P. L. Phys. Rev. Lett. 2005, 95, 1468051-1468054.

(22) Fuhrer, M. S.; Nygard, J.; Shih, L.; Forero, M.; Yoon, Y. G.; Mazzoni, M. S. C.; Choi, H. J.; Ihm, J.; Louie, S. G.; Zettl, A.; McEuen, P. L. Science 2000, 288, 494-497.

(23) Seidel, R. V.; Graham, A. P.; Rajasekharan, B.; Unger, E.; Liebau, M.; Duesberg, G. S.; Kreupl, F.; Hoenlein, W. J. Appl. Phys. 2004, 96, 6694-6699.

(24) Pimparkar, N.; Kumar, S.; Cao, Q.; Rogers, J. A.; Murthy, J. Y.; Alam, M. A. Electron Device Lett. 2007, 28, 157-160.

(25) Stauffer, D.; Aharony, A. Introduction to percolation Theory; Taylor and Francis: London, 1992.

(26) Pike, G. E.; Seager, C. H. Phys. Rev. B 1974, 10, 1421-1434.

(27) Cao, Q.; Hur, S. H.; Zhu, Z. T.; Sun, Y.; Wang, C. J.; Meitl, M. A.; Shim, M.; Rogers, J. A. Adv. Mater. 2006, 18, 304-309.

(28) Pimparkar N.; Guo J.; Alam M. A. IEDM Tech. Digest 2005, 21.5, 541.

(29) Kumar, S.; Pimparkar, N.; Murthy, J. Y.; Alam, M. A. Appl. Phys. Lett. 2006, 88, 123505.

NL062907M 